Check for updates

Cite this: RSC Adv., 2017, 7, 22197

Received 7th March 2017 Accepted 11th April 2017

DOI: $10.1039 / c 7 r a 02767 b$

rsc.li/rsc-advances

\section{PHBV/bioglass composite scaffolds with co- cultures of endothelial cells and bone marrow stromal cells improve vascularization and osteogenesis for bone tissue engineering}

\begin{abstract}
Jun Wu, ab Zhi Wu, ${ }^{c}$ Zhenqiang Xue, ${ }^{\text {ab }}$ Haiyan Li ${ }^{c}$ and Jinbo Liu*ab
Polyhydroxybutyrate-polyhydroxyvalerate (PHBV) and bioglass (BG) have been widely reported to be suitable for bone tissue engineering. However, composite scaffolds with polymers and bioceramics have shown advantages over pure polymer and bioceramic scaffolds for bone tissue engineering. In addition, recent studies have shown that cross-talk between endothelial cells and osteoblastic cells can stimulate bone regeneration compared to tissue engineering constructs containing only one type of cell. Therefore, in this study, we aim to construct an improved engineered bone containing PHBV/BG composite scaffold with co-cultures of human umbilical vein endothelial cells (HUVECS) and human bone marrow stromal cells (HBMSCs) in order to enhance osteogenesis and angiogenesis of bone repair. Results showed that addition of BG into PHBV could enhance osteogenic differentiation of co-cultured HBMSCs and vascularization of co-cultured HUVECs by upregulating paracrine effects between the two types of cells compared to pure PHBV scaffolds. Among all groups, composite scaffolds containing PHBV with 10\% BG showed the strongest stimulatory effects on osteogenic differentiation and vascularization due to their appropriate ion products, specifically, the appropriate concentration of silicon ions. In vivo results also demonstrated that PHBV containing 10\% BG scaffolds with co-cultures of HUVECs and HBMSCs showed the strongest stimulatory effects on osteogenesis and angiogenesis among all groups. Taken together, PHBV/BG scaffolds with co-cultures of endothelial cells and osteogenic cells possess great application potential for bone tissue engineering.
\end{abstract}

\section{Introduction}

Polyhydroxybutyrate-polyhydroxyvalerate (PHBV) has been shown to be a potential biomaterial for tissue engineering as it is biocompatible, biodegradable, and easy to obtain. Besides, its physiochemical properties can be adjusted by tuning the ratio of hydroxyvalerate in the co-polymer PHBV.$^{1,2}$ However, according to the idea of " 3 rd generation bioactive materials", PHBV lacks bioactivity as it does not possess the ability to stimulate cell differentiation and tissue regeneration. ${ }^{3}$ To solve this problem, many bioactive materials have been incorporated into PHBV in order to obtain bioactive composite materials. Bioactive silicate materials, as a type of bioactive material, have been added into PHBV to obtain composite scaffolds with adjustable bioactivity, biodegradability and mechanical properties. ${ }^{3,4}$ For example,

${ }^{a}$ Department of Orthopedics, The Third Affiliated Hospital of Soochow University, 185 Juqian Road, Changzhou, Jiangsu 213003, China. E-mail: czljbljb@126.com

${ }^{b}$ Department of Orthopedics, The First People's Hospital of Changzhou, 185 Juqian Road Changzhou, Changzhou, Jiangsu 213003, China

'Shanghai Jiao Tong University Affiliated Sixth People's Hospital, School of Biomedical Engineering, Shanghai Jiao Tong University, 1954 Huashan Road, Shanghai 200030, China
PHBV/wollastonite composite scaffolds have been demonstrated to be able to induce hydroxyapatite deposition in the scaffolds and adjust the degradation properties of the scaffolds. ${ }^{3}$

For a long time, bioglass (BG) has been demonstrated to be a great biomaterial for bone tissue engineering as it can stimulate osteoblastic differentiation of bone marrow stromal cells and induce calcium deposition of extracellular matrix. ${ }^{\mathbf{5 , 6}}$ Therefore, BG has also been incorporated into various types of polymers, ${ }^{7-10}$ including collagen and polylactic acid, to endow the bioactivity to the composite materials and to obtain scaffolds with improved properties for bone tissue engineering. In addition to bone tissue engineering, our previous study fabricated a composite scaffold PHBV/BG for cartilage tissue engineering. ${ }^{4}$ The rabbit articular chondrocytes were cultured with PHBV scaffolds and PHBV/BG scaffolds with $20 \%$ of BG and we found that the PHBV/BG scaffolds could enhance the adhesion and penetration of chondrocytes into scaffolds and subsequently increase the cartilage tissue synthesis in the scaffolds after the cell/scaffold composites were implanted subcutaneously in nude mice. Interestingly, in the experiment, angiogenesis in the scaffolds were observed in few of PHBV/BG with $20 \%$ BG groups after the scaffolds were implanted in vivo. 
Recently, some literatures reported that silicate ceramics, including BG, can stimulate vascularization of endothelial cells and induce angiogenesis in vitro and in vivo. ${ }^{11-15}$ It has been reported that expression of vascular growth factors in endothelial cells or fibroblasts cultured with 45S5 Bioglass particles, a silicate-based bioglass, or with its dissolution products was upregulated and subsequently improved the vascularization of ECs. ${ }^{5,16,17}$ Since rapid angiogenesis during bone regeneration is one of the critical challenges in bone tissue engineering, ${ }^{18-20}$ according to the previous reports about angiogenic ability of BG, it can be assumed that PHBV/BG composite scaffolds possesses great potential for bone tissue engineering as they can not only stimulate osteogenesis but also enhance angiogenesis on the basis of its adjustable physicochemical properties.

Besides, it has been widely accepted that there is an intimate relationship between bone vascular endothelium and osteoblastic cells (OCs) and interactions between ECs and OCs is the prerequisite for vascularization during bone development. ${ }^{21-25}$ Our previous study demonstrated that, biomaterials, such as silicate bioceramics, could affect the interactions between ECs and OCs, which subsequently enhance osteogenesis and angiogenesis simultaneously. When the scaffolds with cocultures were implanted in vivo, improved osteogenesis and angiogenesis were observed as compared with the same scaffolds with mono-cultured cells. ${ }^{13,26}$ Thus, to design a bone tissue engineering construct with enhanced osteogenesis and angiogenesis ability, PHBV/BG composite scaffolds with co-cultures of ECs and OCs may be a feasible strategy.

Therefore, in this study, we fabricated PHBV/BG scaffolds with different BG content and investigated the effects of scaffolds on vascularization and osteogenic differentiation in cocultures of human umbilical vein endothelial cells (HUVECs) and human bone marrow stromal cells (HBMSCs). Then, optimized PHBV/BG scaffolds seeded with co-cultures of HUVECs and HBMSCs were subcutaneously implanted in nude mouse to further prove the application potential of PHBV/BG scaffolds in stimulating osteogenesis and angiogenesis for bone tissue engineering.

\section{Materials and methods}

\subsection{Preparation and characterization of PHBV and PHBV/BG composite scaffolds}

Poly(3-hydroxybutyrate-co-3-hydroxyvalerate) $\left(\mathrm{PHBV}, \quad M_{\mathrm{w}}=\right.$ 300 000) containing 3 mol\% 3-hydroxyvalerate units was bought from Tianan Biologic Material Co. Ltd. (Ninbo, China). BG particles with size of around $200 \mu \mathrm{m}$ were used to prepare the PHBV/BG composite scaffolds with two different weight ratios of BG : PHBV + 10\% BG and PHBV + 20\% BG by conventional solvent casting-particulate leaching method. ${ }^{27}$ Briefly, PHBV was dissolved in chloroform at a concentration of $10 \%(\mathrm{w} / \mathrm{v})$ with continuous stirring at room temperature. For preparing composite scaffolds, a certain amount of BG powders were added into the PHBV solution and continuous stirring was applied to disperse the BG powders uniformly in the solution. Sodium chloride $(\mathrm{NaCl})$ particles were sieved as porogens with size in the range of $200-300 \mu \mathrm{m}$. After the BG powders were uniformly dispersed in the $\mathrm{PHBV}$ solution, $\mathrm{NaCl}$ particles were incorporated into the PHBV/BG mixed solution or pure PHBV solution. The weight of $\mathrm{NaCl}$ was about 9 times as much as that of PHBV. After being stirred for about $2 \mathrm{~h}$, the suspension of $\mathrm{PHBV} / \mathrm{BG} / \mathrm{NaCl}$ or $\mathrm{PHBV} / \mathrm{NaCl}$ was casted into a Teflon mold with diameter of $60 \mathrm{~mm}$. The samples were then put into fumehood for $24 \mathrm{~h}$ to allow the solvent to evaporate in air followed by vacuum-dry at $60{ }^{\circ} \mathrm{C}$ for $48 \mathrm{~h}$ to remove remaining solvent. Finally, the dried samples were immersed in deionized water to leach out the $\mathrm{NaCl}$ particles in order to create the porous structures of scaffolds. The porous scaffolds were then vacuumdried for future use. Scanning electron microscopy (SEM, FEI, QUANTA 250, Netherlands) was used to observe the porous structure of scaffolds with an accelerating voltage of $10 \mathrm{kV}$.

For testing the compressive strength of scaffolds, scaffolds with the dimension of $10 \mathrm{~mm}$ in diameter and $8-10 \mathrm{~mm}$ in thickness were prepared. The compressive strength testing was conducted with an AG-1 Shimadzu mechanical tester (Shimadzu Co., Japan). The crosshead speed was $0.5 \mathrm{~mm} \mathrm{~min}^{-1}$.

\subsection{Cell isolation and culture}

HUVECs were isolated from human umbilical cord veins according to the method reported previously according to previously described methods ${ }^{28}$ with the consents of the donors of human umbilical cord veins and an Institutional Review Committee of Shanghai Jiao Tong University, school of Biomedical Engineering approved all the protocols. HUVECs were cultured in Endothelial Cell Medium $+5 \%$ FBS (Fetal Bovine Serum) + 1\% ECGS (endothelial cell growth supplement) $+1 \% \mathrm{P} / \mathrm{S}$ (penicillin/streptomycin) (all from Sciencell, USA) (abbreviation in ECM). HBMSCs were purchased from Cyagen Co. Ltd (Guangzhou, China). DMEM (GIBCO) + 10\% FBS (Fetal Bovine Serum) (HyClone) $+1 \% \mathrm{P} / \mathrm{S}$ (penicillin/streptomycin) (abbreviation in DMEM) were used as culture medium of HBMSCs. HUVECs at passages from 3 to 5 and HBMSCs at passages of 2 were used in this study.

For cell experiments, scaffolds with size of $5 \mathrm{~mm}$ (length) $\times 5$ $\mathrm{mm}$ (width) $\times 3 \mathrm{~mm}$ (thickness) were immersed into $75 \%$ ethanol for $2 \mathrm{~h}$, followed by rinse with phosphate buffered saline (PBS). Scaffolds were placed in 24-well plates followed by seeding $5 \times 10^{5}$ HBMSCs only, $5 \times 10^{5}$ HUVECs only or co-cultures of $5 \times$ $10^{5}$ HBMSC and $5 \times 10^{5}$ HUVECs and the cell/scaffold were cultured in $2 \mathrm{ml}$ cell culture medium. The media for co-culture is $1: 1 \mathrm{ECM} / \mathrm{DMEM}$. For the following cell experiments, the cell culture medium was changed every other day.

\subsection{Effects of PHBV/BG scaffolds on vascularization and osteogenic differentiation of cells}

After the cells were cultured within scaffolds for 14 and 28 days, a live-dead viability cytotoxicity kit (Invitrogen) was applied to stain cells and images were taken using a CCD camera (Leica DFC 420C) equipped with a fluorescence microscope (Leica DMI 3000B, Germany) equipped with an excitation filter (490 nm), a dichroic mirror (505 $\mathrm{nm}$ ) and a long-pass emission filter $(530 \mathrm{~nm})$. 
To investigate the effects of PHBV/BG scaffolds on osteogenic differentiation of co-cultured cells, alkaline phosphatase (ALP) expression in cells were evaluated by determining the ALP protein through ALP staining, ALP gene expression and ALP activity. For ALP staining, after the cells were cultured with scaffolds for 14 days, Hank's buffered salt solution (HBSS, GIBCO) was used to wash cells and $4 \%(\mathrm{w} / \mathrm{v})$ paraformaldehyde (PFA) was used to fix cells for $15 \mathrm{~min}$ at room temperature. Thereafter, naphthol/fastblue ready-to-use solution (naphthol/ fastblue $=166 \mu \mathrm{l} / 4 \mathrm{ml}$, Sigma) was used to incubate the cells/ scaffolds at $37{ }^{\circ} \mathrm{C}$ for $20 \mathrm{~min}$. At the end of the incubation, cells/scaffolds were washed with HBSS again to remove the excess stain solution. Then, the samples were put on glass slides for observation with an inverted microscope and images were taken by a CCD camera. In addition, an alkaline phosphatase assay kit (abcam) was used to detecting ALP activity of cells in the scaffolds. In the kit, $p$-nitrophenyl phosphate (pNPP) is used as a phosphatase substrate. As pNPP can be dephosphorylated by ALP and dephosphorylated pNPP turns yellow, the ALP activity can be calculated by detecting the dephosphorylated pNPP at $405 \mathrm{~nm}$ and can be expressed in $\mathrm{nmol} \mathrm{ml} \mathrm{min}^{-1}$.

\subsection{Expression of VEGF, KDR, BMP-2 and ALP in mono- cultured or co-cultured cells within scaffolds}

After the cells were cultured with scaffolds for 14 days, the scaffolds were gently smashed into small pieces and cells were trypisinized. Cells were then collected by centrifugation. To detect the gene expression of critical molecules in co-cultured cells, magnetic beads functionalized with CD31 antibody, a specific protein of endothelial cells (Invitrogen) was used to separate HUVECs from HBMSCs according to the method established by Guillotin et al. ${ }^{21}$ Briefly, at the predetermined time point, co-cultured cells were detached by trypsinization. Cell pellets were obtained by centrifuging cell suspension followed by re-suspending the cells in HBSS in a centrifuge tube. Then, solution containing magnetic beads were added into the cell suspension at certain concentration and the mixture was incubated for $30 \mathrm{~min}$ with agitation at $4{ }^{\circ} \mathrm{C}$. After that, the centrifuge tube containing the mixture was placed on a magnet stack for $5 \mathrm{~min}$. During this process, the HUVECs connected with the magnetic beads adhered and collected on the tube wall near the magnet stack. Thus, the HUVECs were separated from the HBMSCs in the supernatant. Co-HUVEC and co-HBMSC referred to the separated co-culture HUVECs and co-cultured HBMSCs, respectively.

After the cells were ready, gene expression of VEGF, KDR, BMP-2 and ALP from the cells cultured was detected by Quantitative real-time polymerase chain reaction (Q-RT-PCR). E. Z. N. A Total RNA kit I (OMEGA, Bio-tek) was used to extract RNA after the cells were washed twice with cold PBS. A nanodrop 1000 reader (Thermo SCIENTIFIC) was used to measure the concentration of RNA. After obtaining RNA, a ReverTra Ace-a kit (Toyobo Co., Ltd, Japan) cDNA was used to synthesize cDNA according to the manufacture's instruction. Then, the cDNA was diluted with sterilized Mill-Q water and $1 \mathrm{ml}$ of diluted CDNA was mixed with $9 \mathrm{ml}$ SYBR-Green Master Mix (ToYOBO Co.,
Ltd), primer of VEGF, or KDR, or BMP-2 or ALP or osteocalcin (OCN) (all from Sangon Bioech (Shanghai) Co., Ltd) in the final concentration of $400 \mathrm{nM}$. GAPDH (glyceraldehyde 3-phosphate dehydrogenase) was used as a housekeeping gene. The sequences for VEGF, KDR, BMP-2, ALP, OCN and GAPDH are listed in Table 1 . The mixture was then loaded in a 384-well plate and real-time PCR analysis was performed using the 7900 real-time PCR system (Applied Biosystems) with the process of denaturation for $1 \mathrm{~min}$ at $95{ }^{\circ} \mathrm{C}$ and 40 cycles $\left(95^{\circ} \mathrm{C}\right.$ for $15 \mathrm{~s}$, $60{ }^{\circ} \mathrm{C}$ for $15 \mathrm{~s}, 72{ }^{\circ} \mathrm{C}$ for $45 \mathrm{~s}$ ). Triplicates were prepared for each sample. At the end of PCR, SDS 2.3 software was used to analyze the data and $\Delta \Delta C t$ method was used to compare data. Each data was first normalized to GAPDH gene expression of each condition and then quantified relative to the corresponding gene expressions of control samples (standardized to 1).

\subsection{Ion productions of scaffolds}

The ion extracts of scaffolds were prepared according to the procedures adapted from literatures and ISO10993-1. ${ }^{\mathbf{1 1}}$ Briefly, one PHBV, PHBV $+10 \%$ BG or PHBV $+20 \%$ BG scaffold was immersed in $2 \mathrm{ml}$ DMEM/ECM $=1: 1$ cell culture medium and incubated in a humidified $37{ }^{\circ} \mathrm{C} / 5 \% \mathrm{CO}_{2}$ incubator for $48 \mathrm{~h}$. Then, the supernatant was collected and concentrations of $\mathrm{Ca}$, Si and $\mathrm{P}$ ions were detected by inductively coupled plasma atomic emission spectroscopy (ICP-AES; Optima 3000DV, Perkin Elmer, USA).

\subsection{In vivo evaluation of angiogenesis and osteogenesis of cell/scaffolds}

2.6.1 Implantation of cells/scaffolds. The experiment was performed in Shanghai Jiao Tong University and an Institutional Review Committee of Shanghai Jiao Tong University, School of Biomedical Engineering approved all animal study protocols. All experiments comply with the current laws of China. Pockets were created on dorsal of 4 week old male mice (BALB/c) according to previous methods for implanting scaffolds with cells. ${ }^{13}$ Briefly, a $2 \times 2 \mathrm{~cm}$ area of the mouse back was shaved and wiped with gauze soaked with $75 \%$ ethanol after the mice were anesthetized with an intraperitoneal injection of chloral hydrate $\left(350 \mathrm{mg} \mathrm{kg}^{-1}\right)$. Then, the centre of the shaved

Table 1 Primer sequences used in quantitative real-time polymerase chain reaction

\begin{tabular}{|c|c|c|}
\hline Gene & Primer sequences & $T_{\mathrm{m}}\left({ }^{\circ} \mathrm{C}\right)$ \\
\hline VEGF $_{165}$ & $\begin{array}{l}\text { F:5' TGCGGATCAAACCTCACCA } 3^{\prime} \\
\text { R:5' CAGGGATTTTTCTTGTCTTGCT } 3^{\prime}\end{array}$ & 58 \\
\hline KDR & $\begin{array}{l}\text { F:5' GTGATCGGAAATGACACTGGAG } 3^{\prime} \\
\text { R:5' CATGTTGGTCACTAACAGAAGCA } 3^{\prime}\end{array}$ & 60 \\
\hline ALP & $\begin{array}{l}\text { F:5' AGCCCTTCACTGCCATCCTGT } 3^{\prime} \\
\text { R:5' ATTCTCTCGTTCACCGCCCAC } 3^{\prime}\end{array}$ & 58 \\
\hline BMP-2 & $\begin{array}{l}\text { F:5' AACACTGTGCGCAGCTTCC } 3^{\prime} \\
\text { R:5' CTCCGGGTTGTTTTCCCAC } 3^{\prime}\end{array}$ & 63 \\
\hline OCN & $\begin{array}{l}\text { F:5' TCACACTCCTCGCCCTATTGG } 3^{\prime} \\
\text { R:5' TACCTCGCTGCCCTCCTGCTT } 3^{\prime}\end{array}$ & 62 \\
\hline GAPDH & $\begin{array}{l}\text { F:5' GATTTGGTCGTATTGGGCG } 3^{\prime} \\
\text { R:5' CTGGAAGATGGTGATGG } 3^{\prime}\end{array}$ & 60 \\
\hline
\end{tabular}


skin was cut into a small longitudinal incision and the subcutaneous facial tissue was dissected. Then, pockets were created under the skin (between the skin and muscle layers). 4 pockets were made for implanting 4 different implants on each mouse.

After the cells were cultured in scaffolds for 14 days in vitro, cells with scaffolds were subcutaneously implanted into the pockets. For 8 different conditions (PHBV, PHBV with HBMSCs, PHBV with HUVECs, PHBV with co-cultured HUVECs and HBMSCs, PHBV/BG, PHBV/BG with HBMSCs, PHBV/BG with HUVECs, PHBV/BG with co-cultured HUVECs and HBMSC) at each time point, 6 mice for total 24 implants were needed. For whole animal experiment, 24 mice were needed. After the scaffolds with or without cells were implanted into the pockets, the incisions were sutured and the wounds were covered with tissue adhesives. Animals were killed at determined time points and implants were analyzed for vascularization or osteogenesis.

2.6.2 Tissue preparation and histological staining. The specimens obtained at different time points were fixed in $10 \%$ formalin for 4-6 h, embedded in paraffin, cut into $5 \mu \mathrm{m}$ sections longitudinally and the sections were collected on glass slides. To visualize the overall tissue morphology, the sections were stained with Gill's 3 hematoxylin and aqueous eosin Y solution (Sigma-Aldrich) (H\&E). Von Kossa staining was performed according to kit manufactures' instruction (Sigma-Aldrich). Human-derived blood vessels and human bone sialoprotein (BSP) were detected using a specific anti-human CD31 antibody and anti-human BSP antibody, respectively (Invitrogen). The stained slides were finally mounted, and covered with coverslips.

2.6.3 Quantification of blood vessels and osteogenesis. Five samples from each condition were observed and photographed with an upright fluorescence microscope (Leica DM 4000, German). Blood vessels were identified in H\&E-stained tissues by defined lumens containing red blood cells. Total number of blood vessels in the implants were counted manually. Human-derived blood vessels were identified according to the staining of human CD31 antigen and their numbers were counted from images taken at $200 \times$ magnification. Osteogenic differentiation of HBMSCs and mineralization of extracellular matrix synthesized by HBMSCs in the scaffolds were determined by BSP staining and von Kossa staining, respectively. Images were taken. BSP positive staining is blackbrown and von Kossa positive staining area is dark in images.

\subsection{Statistical analysis}

Data were expressed as means \pm standard deviation (SD). A oneway analysis of variance (ANOVA) with Tukey's post hoc test was used for statistical analysis of multiple comparisons. Significant difference were considered when $p<0.01$ (** or \#).

\section{Results}

\subsection{Characterization of scaffolds}

SEM images of surface and cross-section of scaffolds are shown in Fig. 1. It can be seen that all scaffolds were porous with pore size in a range of 200-400 $\mu \mathrm{m}$. After the scaffolds were immersed in cell culture media, the concentrations of $\mathrm{Ca}, \mathrm{P}$ and Si ions in the cell culture media are shown in Table 2. Interestingly, the concentrations of $\mathrm{Ca}$ and $\mathrm{P}$ in different media were almost same while the concentration of $\mathrm{Si}$ ions in media soaking PHBV/BG composite scaffolds with different content of BG $\left(1.32 \mu \mathrm{g} \mathrm{ml}^{-1}\right.$ for PHBV $+10 \% \mathrm{BG}$ and $2.72 \mu \mathrm{g} \mathrm{ml}^{-1}$ for PHBV + $20 \% \mathrm{BG}$ ) was significantly higher than that in DMEM, ECM, DMEM + ECM and DMEM + ECM soaking PHBV scaffolds (around $0.36 \mu \mathrm{g} \mathrm{ml}^{-1}$ ).

The compressive strength of scaffolds is $0.15 \pm 0.02 \mathrm{MPa}$, $0.25 \pm 0.04 \mathrm{MPa}$ and $0.32 \pm 0.03 \mathrm{MPa}$ for pure PHBV, PHBV + $10 \%$ BG and PHBV $+20 \%$ BG scaffolds, respectively. The compressive strengths of $\mathrm{PHBV} / \mathrm{BG}$ composite scaffolds were
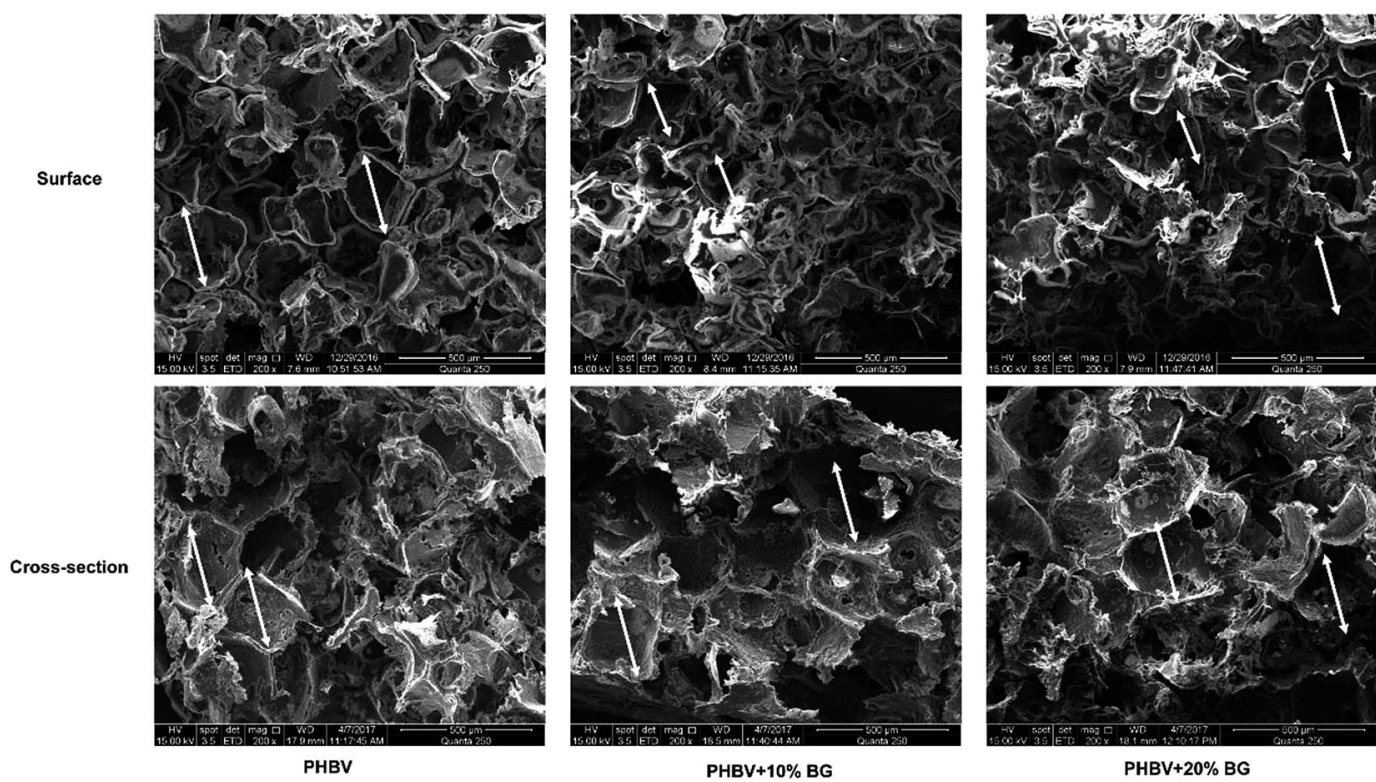

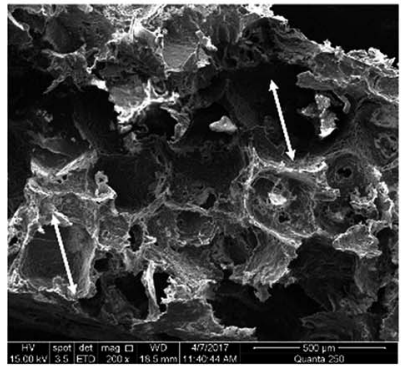

PHBV+10\% BG

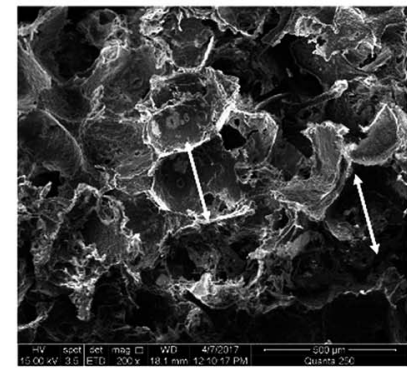

$\mathrm{PHBV}+20 \% \mathrm{BG}$

Fig. 1 SEM images of PHBV, PHBV + 10\% BG and PHBV + 20\% BG scaffolds. 
Table 2 Ion concentration of cell culture media and scaffold extracts

\begin{tabular}{lrrr}
\hline & $\mathrm{Ca}\left(\mu \mathrm{g} \mathrm{ml}^{-1}\right)$ & $\mathrm{P}\left(\mu \mathrm{g} \mathrm{ml}^{-1}\right)$ & $\mathrm{Si}\left(\mu \mathrm{g} \mathrm{ml}^{-1}\right)$ \\
\hline (1) DMEM & $63.32 \pm 0.24$ & $17.09 \pm 0.25$ & $0.35 \pm 0.04$ \\
(2) ECM & $61.25 \pm 0.23$ & $17.35 \pm 0.28$ & $0.36 \pm 0.03$ \\
(1) + (2) $=1: 1$ & $62.45 \pm 0.43$ & $17.18 \pm 0.17$ & $0.34 \pm 0.05$ \\
PHBV in (1) + (2) & $63.82 \pm 0.24$ & $17.11 \pm 0.18$ & $0.35 \pm 0.02$ \\
PHBV + 10\% BG in (1) + (2) & $70.20 \pm 0.73$ & $16.81 \pm 0.22$ & $1.32 \pm 0.12^{a}$ \\
PHBV + 20\% BG in (1) + (2) & $71.14 \pm 0.68$ & $16.77 \pm 0.34$ & $2.72 \pm 0.22^{a}$ \\
a Indicates that the silicon ion concentration in the samples is \\
significantly higher than those in DMEM, ECM and DMEM + ECM (p \\
$<0.01)$.
\end{tabular}

significantly higher than that of pure PHBV scaffolds. However, the compressive strength of PHBV $+10 \%$ BG and PHBV $+20 \%$ BG scaffolds showed no significant difference.

\subsection{PHBV/BG composite scaffolds stimulated vascularization of co-cultures of HUVECs and HBMSCs in vitro}

After the cells were cultured in the different scaffolds for 14 and 28 days, live-dead assay was used to stain the cells in order to facilitate the observation of cells in the scaffolds. It can be seen that the cells grew very well in PHBV/BG scaffolds (Fig. 2a). Incorporation of BG in scaffolds supported the HUVEC adhesion and proliferation as more HUVECs were observed in the composite scaffolds than those on pure PHBV scaffolds at 14 days. In addition, composite scaffolds with co-cultures facilitated the vascularization of co-cultured HUVECs (co-HUVEC) as compared to the pure PHBV scaffolds as more cell self-assemble and capillary-like networks (white arrows) were observed within the composite scaffolds (Fig. 2a). However, either pure PHBV or PHBV/BG composite scaffolds could not support the vascularization when there was only mono-cultured HUVECs or HBMSCs.

In addition, when the HUVECs and HBMSCs were cocultured in scaffolds for 14 days, typical paracrine effects were observed as it was co-HBMSCs that mainly expressed VEGF and subsequently co-HUVECs took charge of KDR expression. When different scaffolds were compared, the composite scaffolds significantly stimulated VEGF gene expression from coHBMSCs and KDR gene expression from co-HUVECs although the mono-cultured cells were also stimulated (Fig. 2b and c). Among all composite scaffolds, PHBV $+10 \%$ BG scaffolds showed highest stimulatory effects on VEGF gene expression from co-HBMSCs and KDR gene expression from co-HUVECs, suggesting that $10 \%$ wt was the optimal content of $\mathrm{BG}$ for stimulating vascularization.

\subsection{PHBV/BG composite scaffolds stimulated osteogenic differentiation of co-cultures of HUVECs and HBMSCs in vitro}

Fig. 3 shows the osteogenic differentiation of HBMSCs in monocultured and co-cultures. ALP staining, ALP activity and ALP gene expression were showed in Fig. 3a-c, respectively. Results show that the incorporation of BG in the PHBV scaffolds enhanced the osteogenic differentiation of co-HMBSCs. In addition, the co-HBMSC in PHBV $+10 \%$ BG composite scaffolds possessed highest osteogenic differentiation among all groups. In addition to ALP expression, BMP-2 gene expression was determined in cells cultured under different conditions and results were shown in Fig. 3d. Interestingly, paracrine effects

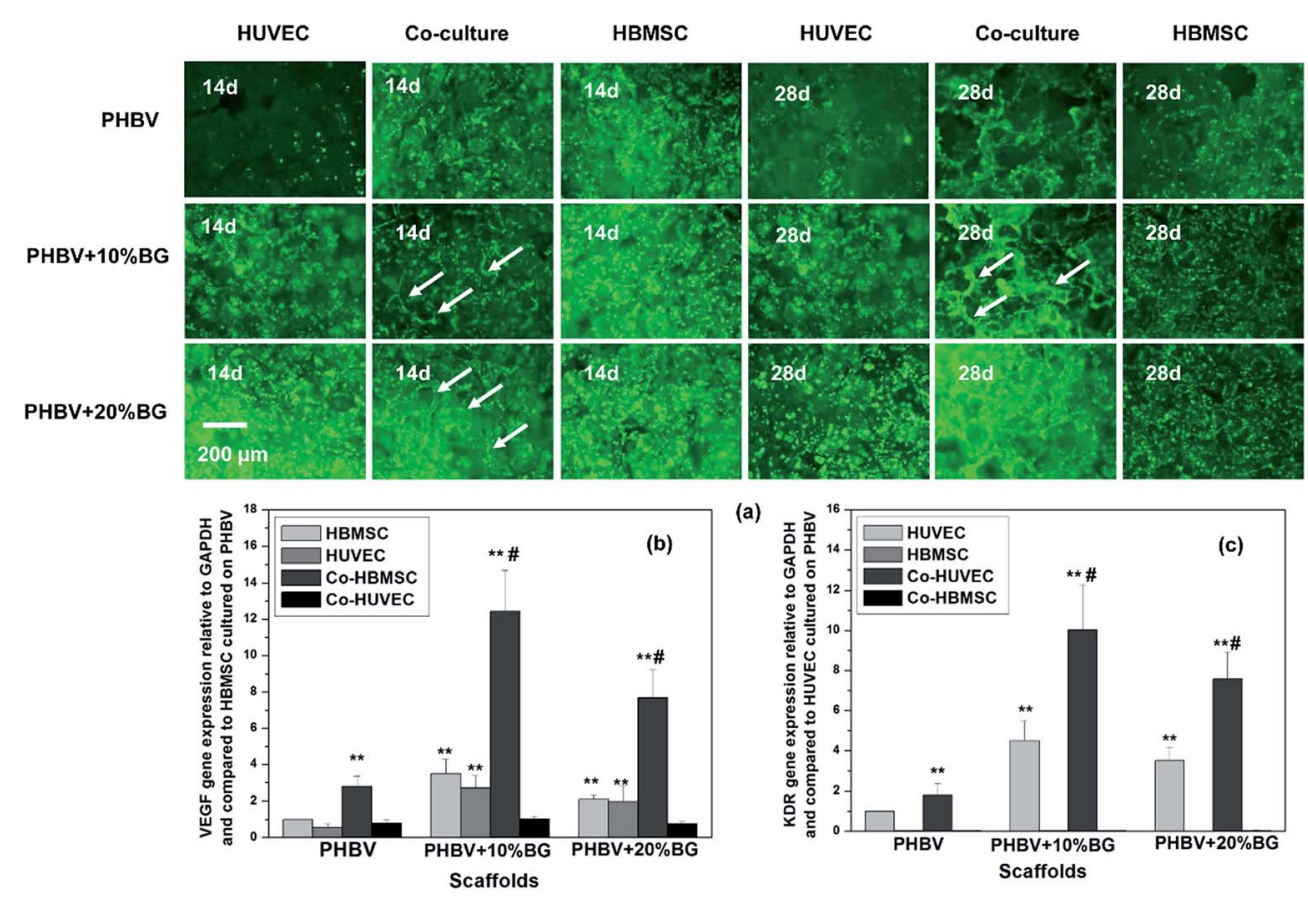

Fig. 2 Effects of different scaffolds on vascularization of cells. (a) live-dead staining of cells cultured in different scaffolds for 14 and 28 days. (b) VEGF gene expression in cells cultured in different scaffolds for 14 days. (c) KDR gene expression in cells cultured in different scaffolds for 14 days. 


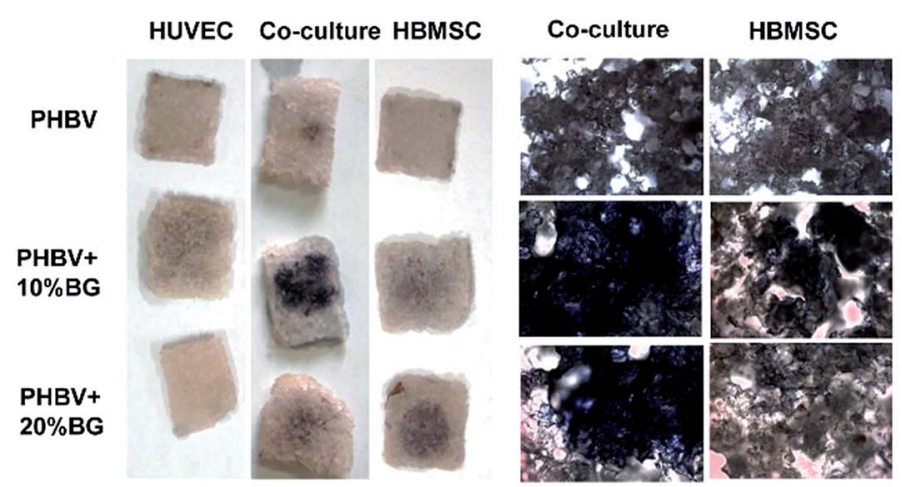

(a)

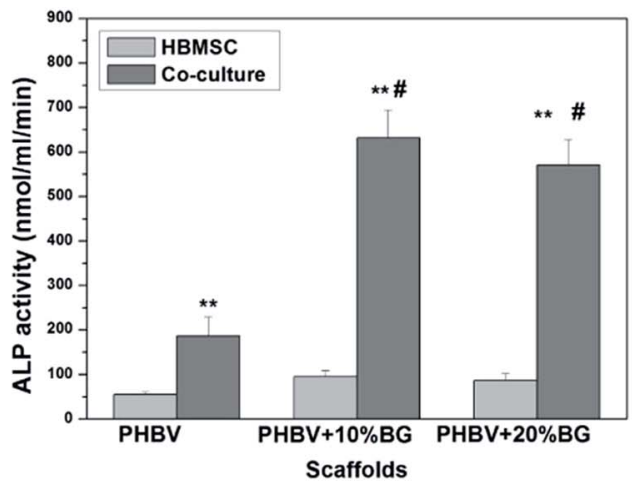

(b)

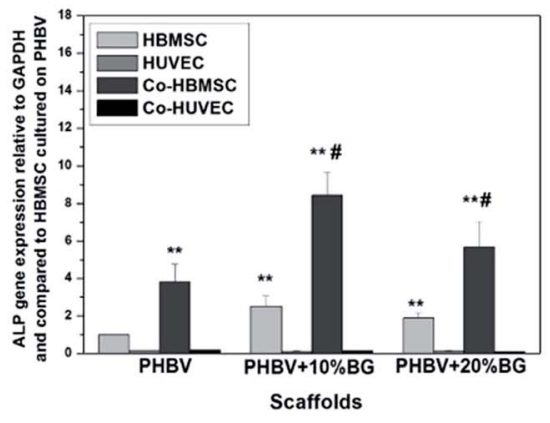

(c)

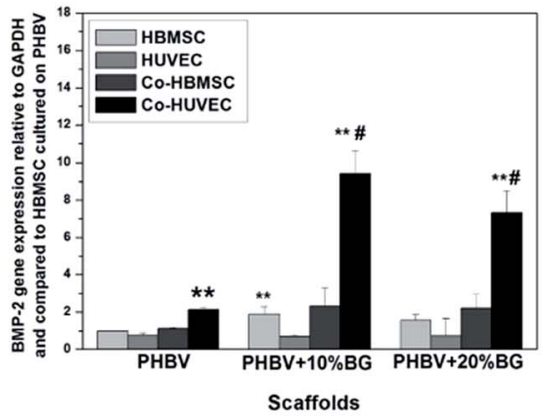

(d)

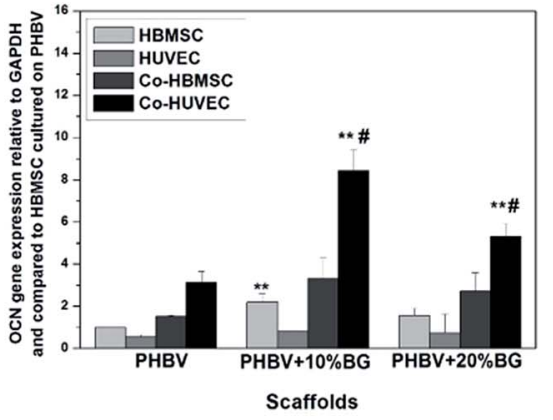

(e)

Fig. 3 Effects of different scaffolds on osteogenic differentiation of cells. (a) ALP staining of cells cultured in different scaffolds for 14 days. (b) ALP activity of cells cultured in different scaffolds for 14 days. (c) ALP gene expression of cells cultured in different scaffolds for 14 days. (d) BMP-2 gene expression of cells cultured in different scaffolds for 14 days. (e) OCN gene expression of cells cultured in different scaffolds for 14 days.

were also observed in co-cultures as BMP-2 was mainly expressed by co-HUVECs but not by co-HBMSCs. In addition, BG in the composite scaffolds strongly stimulated the BMP-2 expression from co-HUVECs and composite scaffolds containing $10 \%$ BG showed the highest stimulatory effects on coHUVECs among all scaffolds. The gene expression of OCN also indicated that there were paracrine effects between coHUVECs and co-HBMSCs as OCN was mainly expressed by coHUVECs. Addition of BG in the scaffolds stimulated the OCN expression in co-HUVECs and co-HUVECs in PHBV $+10 \% \mathrm{BG}$ scaffolds showed the highest OCN expression, which is similar to that of BMP-2 (Fig. 3e). Considering the strong stimulatory effects of $\mathrm{PHBV}+10 \% \mathrm{BG}$ on vascularization and osteogenic differentiation, PHBV and PHBV $+10 \%$ BG scaffolds (abbreviated as $\mathrm{PHBV} / \mathrm{BG}$ ) for further animal tests.

\subsection{PHBV/BG composite scaffolds with co-cultures stimulated vascularization and angiogenesis in vivo}

HE staining images of PHBV and PHBV/BG scaffolds with cells after 2 and 6 week implantation are shown in Fig. 4. The scaffolds were marked by "S". Red blood cells (arrows) could be clearly observed. Neovascularization within different scaffolds was analyzed by counting blood vessels that contained red blood cells inside. It can be seen that, in different scaffolds with same cell condition, more red blood cells were observed in PHBV/BG scaffolds than in PHBV scaffolds, which indicated that BG could stimulate the neovascularization at both time points. In addition, in same scaffolds with different cell situations, more blood vessels were observed in scaffolds containing co-cultures of HBMSCs and HUVECs. Furthermore, with time increased, more blood vessels could be found in PHBV/BG composite scaffolds with co-cultures at week 6 than those at week 2. Therefore, there were most of blood vessels in PHBV/BG composite scaffolds with co-cultures of HBMSC and HUVECs among all groups.

To further identify blood vessels formed by HUVECs cotransplanted with HBMSC in scaffolds, blood vessels were stained with CD 31 interacting with human endothelial cells. In Fig. 5, the human-derived vessels were pointed out by arrowheads, which were brown structures. At both 2 and 6 weeks implantation, in different scaffolds with same cell situation, more brown structures were observed in the PHBV/BG scaffolds than those in PHBV scaffolds. In addition, at both 2 and 6 weeks implantation, in same scaffolds with different cell situations, more brown structures were observed in scaffolds with cocultures as compared to those in the same scaffolds with HBMSCs or HUVECs. Furthermore, as compared to same situation at week 2, more blood vessels were formed at week 6 . All these results indicated that the combination of $\mathrm{PHBV} / \mathrm{BG}$ 

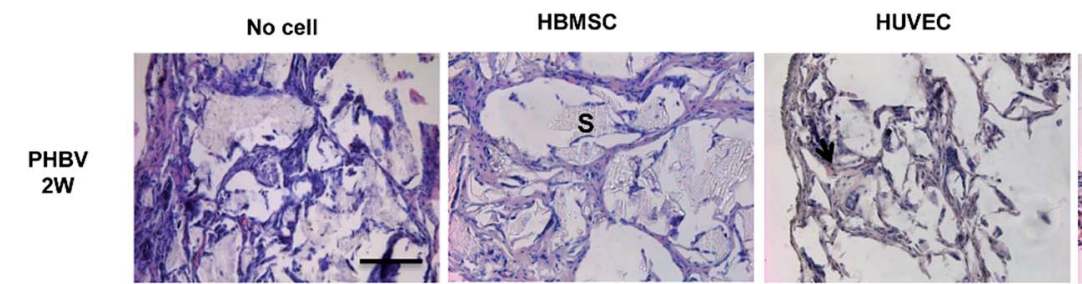

HUVEC+HBMSC
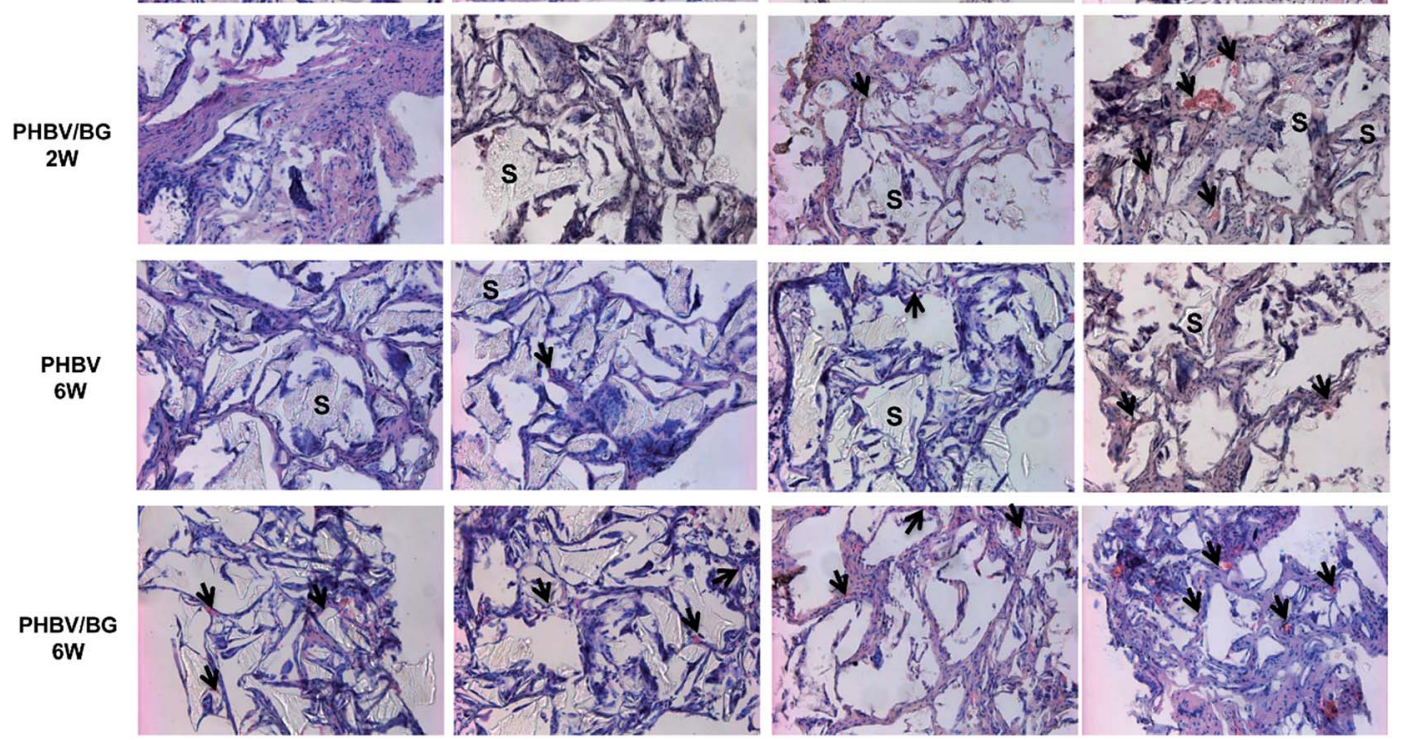

Fig. $4 \mathrm{H}$ HE-stained sections of different implants at 2 and 6 weeks. Functional blood vessels were defined by structures that had a clearly defined lumen containing red blood cells (arrows). Bar $=50 \mu \mathrm{m}$.
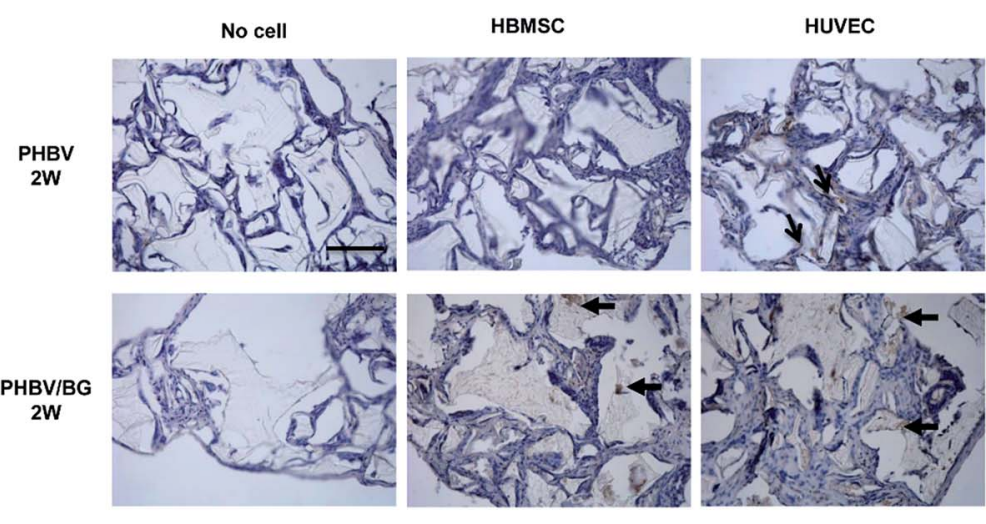

HUVEC+HBMSC
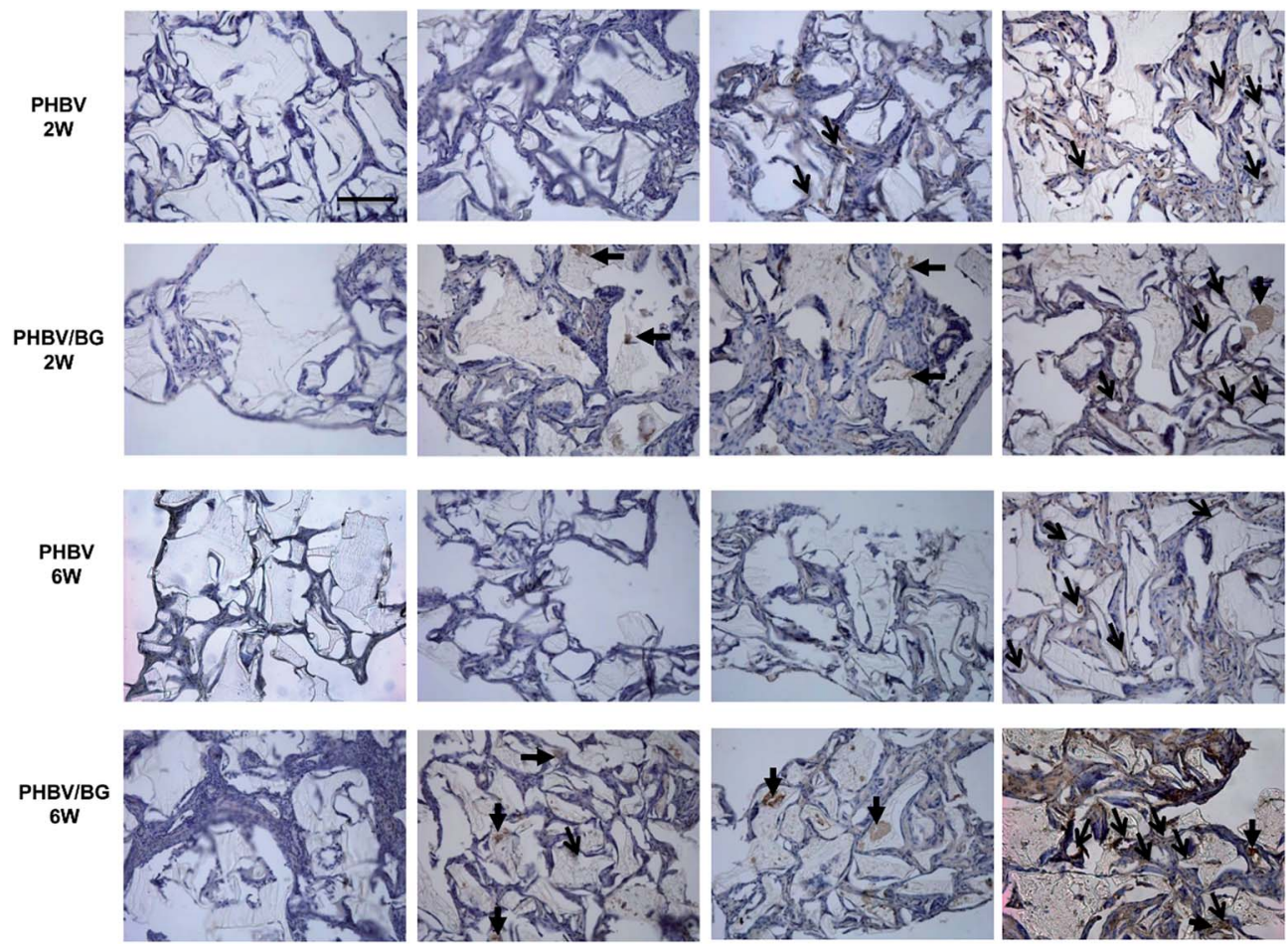

Fig. 5 Human-derived vessels in different implants at 2 and 6 weeks were detected via human CD31 immunostaining (brown structures, arrows). Bar $=50 \mu \mathrm{m}$. 
composite scaffolds with co-culture of HUVECs and HBMSCs system could stimulate the neovascularization derived from transplanted HUVECs.

\subsection{PHBV/BG composite scaffolds with co-cultures stimulated osteogenic differentiation in vivo}

Osteogenic differentiation in scaffolds with or without cells were determined by BSP immunostaining. Osteoblasts secreted BSP attached on ECM and stained in dark brown-black (arrows in Fig. 6). It can be seen from the images that very few BSP was observed in scaffolds with HBMSC alone and HUVEC alone and scaffolds without cells. After being implanted for 4 and 8 weeks, in different scaffolds with co-cultures, more BSP was obviously observed in PHBV/BG scaffolds than in PHBV scaffolds, which indicated that BG in composite scaffolds could stimulate the osteogenic differentiation of co-HBMSCs in the co-cultures. In addition, in same scaffolds with different cell situations, more BSP was observed in scaffolds with co-cultures than that in scaffolds with mono-cultured cells. Therefore, there was most BSP in PHBV/BG composite scaffolds with co-cultures.

Mineralization of ECM in implants was further indicated by von Kossa staining (black regions in Fig. 7). After being implanted for 4 and 8 weeks, in different scaffolds with same cell situation, more mineralized matrix was formed in PHBV/BG composite scaffolds than that in PHBV scaffolds. In addition, in same scaffolds with different cell situation, more mineralized ECM was observed in scaffolds with co-cultures of HBMSCs and HUVECs than that in same scaffolds with mono-cultured cells.
Among all groups, strongest mineralization was observed in PHBV/BG scaffolds with co-cultures.

\section{Discussion}

Vascularization is very critical for enhancing bone tissue engineering as it is difficult for blood vessel growing into engineered bone constructs larger than a few millimeters in volume. ${ }^{\mathbf{1 9 , 2 0 , 2 9}}$ To obtain scaffolds for bone tissue engineering, in this study, the effects of composite scaffolds containing PHBV and BG on vascularization and osteogenic differentiation were evaluated in vitro and in vivo. Results showed that the $\mathrm{PHBV} / \mathrm{BG}$ scaffolds containing $10 \%$ BG could stimulate vascularization of HUVECs and osteogenic differentiation of HBMSCs in a direct contact coculture of HUVECs-HBMSCs. After the composite scaffolds with co-cultures were implanted subcutaneously, blood vessels ingrowth from surrounding tissue and formed by loaded coHUVECs were found in the composite scaffolds. In addition, osteogenic differentiation of co-HBMSCs in the scaffolds was observed, together with mineralization of ECM. Therefore, the PHBV/BG scaffolds with $10 \%$ BG show great application for bone tissue engineering as they can stimulate angiogenesis/ vascularization and osteogenesis simultaneously.

Both PHBV and BG have been widely reported to be suitable

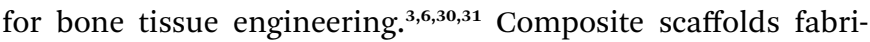
cated from polymers and bioceramics can take advantages of polymer and bioceramics, such as biodegradability, biocompatibility and elasticity of polymer as well as bioactivity of bioceramics, which can make up the disadvantages of pure
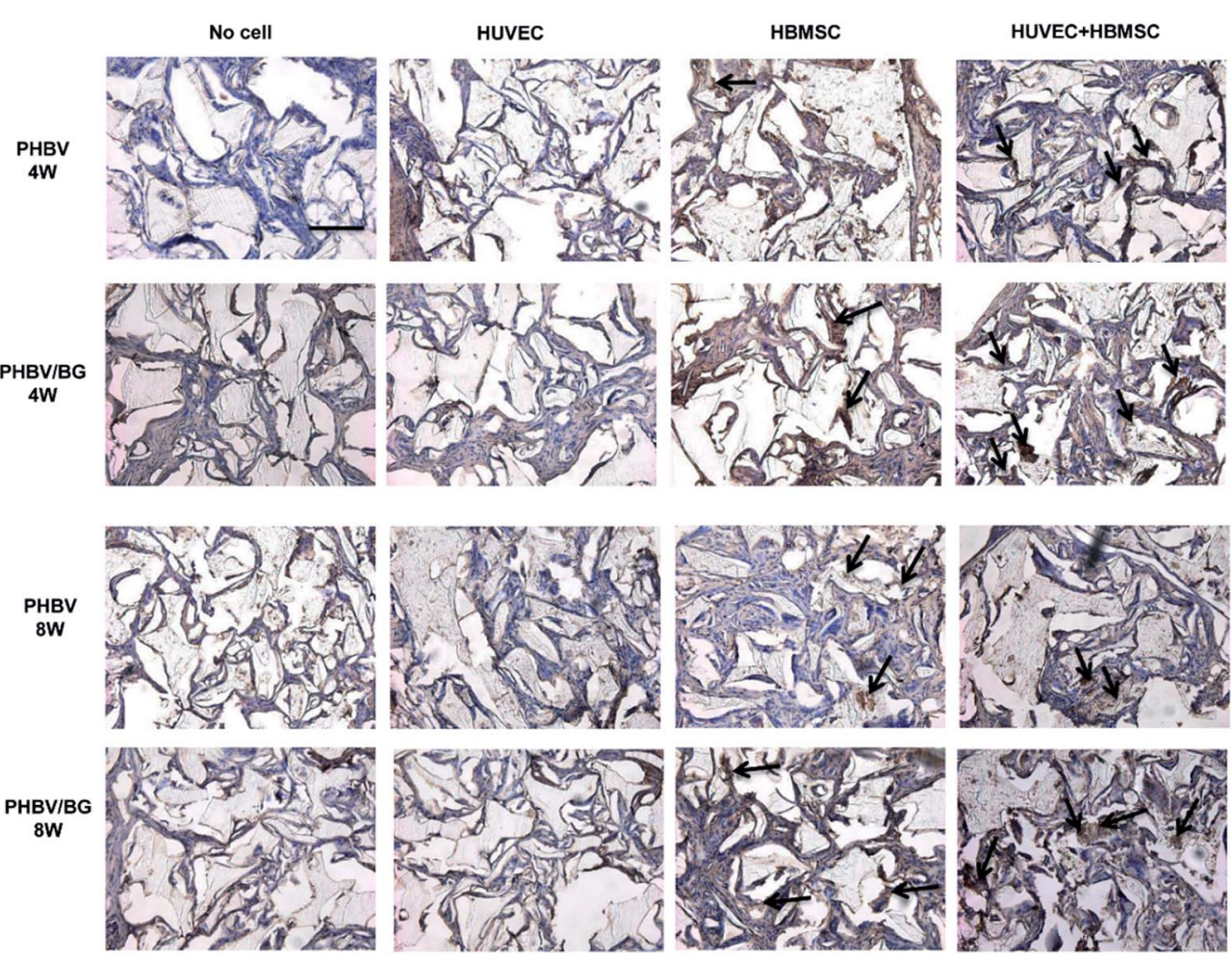

Fig. 6 BSP secreted by osteoblasts in ECM was detected by immunostaining (dark browne black stain, arrows) after 4 and 8 weeks postimplantation. Bar $=50 \mu \mathrm{m}$. 

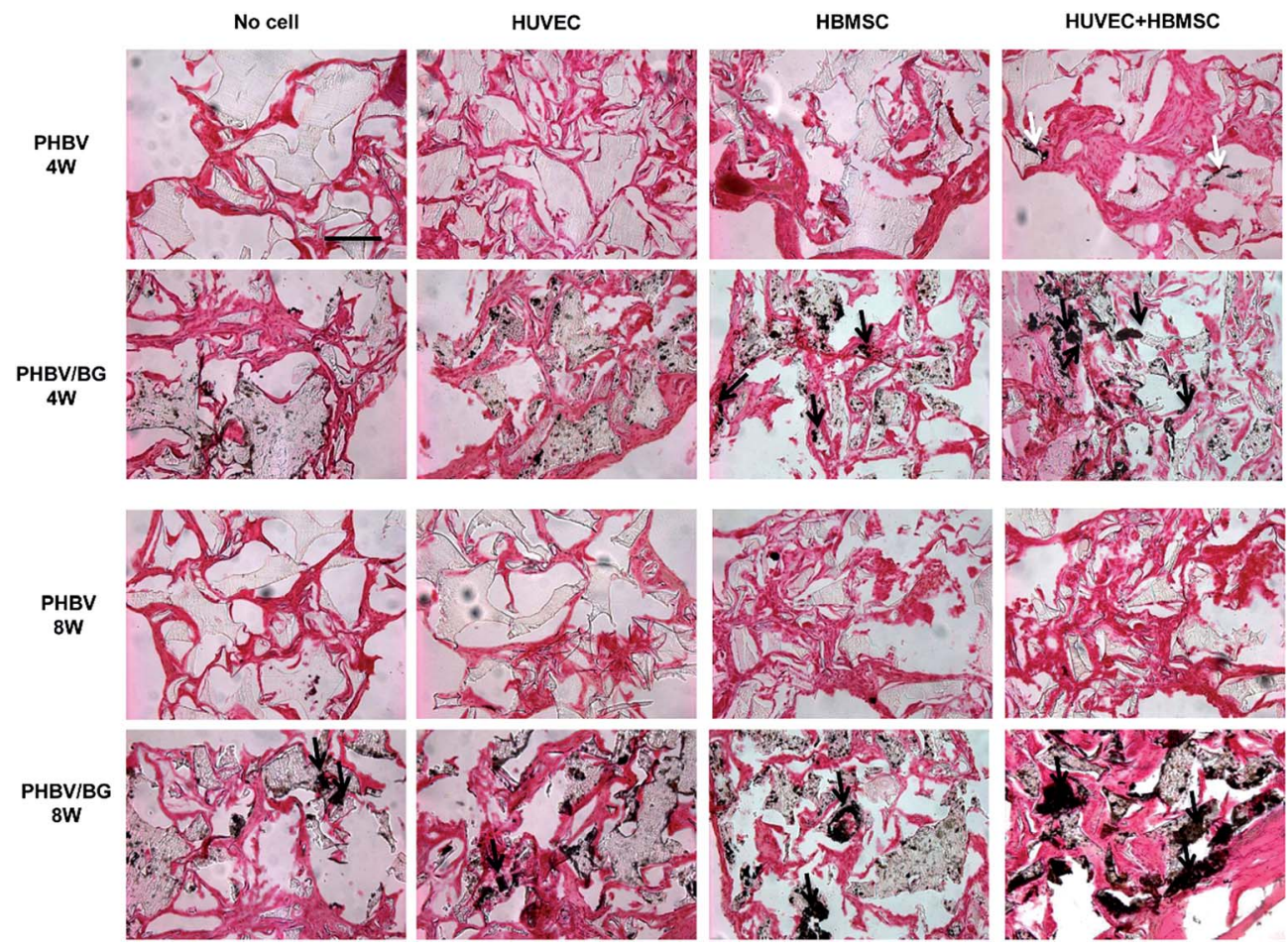

Fig. 7 Mineralized matrix in different implants at 4 and 8 weeks post-implantation was indicated by von Kossa staining (black regions, arrows). Bar $=50 \mu \mathrm{m}$.

polymer or bioceramics, such as lack of bioactivity, acidic biodegradable products of polymer and brittleness of bioceramics. ${ }^{3,27,32,33}$ In addition, the physicochemical and bioactivity properties of composite scaffolds are easily to be controlled through adjusting the component of the scaffolds. In this study, the bioactivity of the PHBV/BG composite scaffolds was adjusted by changing the amount of the BG in the scaffolds. All results indicate that composite scaffolds with 10\% BG are the best components for stimulating vascularization and osteogenesis. For a long term, ionic products of BG have been widely reported to have stimulatory effect on osteogenic differentiation. Recently, ionic products of BG and other silicate bioceramics, specifically, the silicon ions with concentration in the range of $0.7-1.8 \mu \mathrm{g} \mathrm{ml}^{-1}$, have been demonstrated to be able to play an important role in stimulating vascularization of endothelial cells. ${ }^{11-13,34}$ In this study, the concentrations of silicon ions in the cell culture media of $\mathrm{PHBV} / 10 \%$ BG composite scaffolds followed in the range, which indicated that this scaffold had best stimulatory effect among all groups.

During development of most tissue or organs, cell-cell interactions play critical roles. ${ }^{24}$ Specifically, for bone tissue engineering, there are intimate relationships between ECs and OCs. ${ }^{22,23,35,36} \mathrm{Li}$ et al. reported that poly(lactic-co-glycolic acid)/ calcium silicate (PLGA/CS) composite scaffolds loaded with co-cultures of ECs and BMSCs showed higher level of osteogenic differentiation and vascularization as compared to same scaffolds with mono-cultured cells. ${ }^{13}$ In this study, same results have been obtained. The co-cultures of HUVECs and HBMSCs in scaffolds showed stronger vascularization and osteogenesis as compared to mono-cultured cells in same scaffolds. In vitro results indicated that the paracrine effects between HUVECs and HBMSCs were upregulated, which resulted in more VEGF were secreted in co-HBMSCs and more KDR were activated in co-HUVECs as compared to those in mono-cultured cells with same scaffolds. For the mechanism, Li et al. has indicated that KDR activation might initiate angiogenesis pathway and osteogenesis pathway simultaneously. ${ }^{13}$ Therefore, the upregulation of PHBV/BG scaffolds on paracrine effects between HUVECs and HBMSCs may result in the enhanced vascularization and osteogenic differentiation, which indicates that the tissue engineering construct containing PHBV/BG scaffolds and co-culture of HUVECs and HBMSCs possesses great application potential for bone tissue engineering.

\section{Conclusion}

In this study, we aimed to design a tissue engineering construct for bone tissue engineering. PHBV/BG composite scaffolds were fabricated and co-cultures of HUVECs and HBMSCs were used as seeding cells. As compared to pure PHBV scaffolds, the composite scaffolds could stimulate the vascularization and osteogenic differentiation of co-cultures and the PHBV $+10 \%$ BG showed the strongest stimulatory effects among all groups in vitro and in vivo. Proper ion products released by BG in the composite scaffolds may enhanced paracrine effects between the two kinds of cells, which results in the stimulatory effects of PHBV $+10 \%$ BG on angiogenesis and osteogenesis. 


\section{Ethical standards}

The authors declare that all of the experiments comply with the current laws of China.

\section{Acknowledgements}

This work was supported by Changzhou Science and Technology Plan Project (Grant No. CJ20159016) and Natural Science Foundation of China (Grant No. 31470918).

\section{References}

1 J. Y. Sun, J. Wu, H. Y. Li and J. Chang, Macroporous poly(3hydroxybutyrate-co-3-hydroxyvalerate) matrices for cartilage tissue engineering, Eur. Polym. J., 2005, 41, 2443-2449.

2 H. W. Tong, M. Wang and W. W. Lu, Electrospinning and evaluation of PHBV-based tissue engineering scaffolds with different fibre diameters, surface topography and compositions, J. Biomater. Sci., Polym. Ed., 2012, 23, 779-806.

$3 \mathrm{H}$. Li and J. Chang, Fabrication and characterization of bioactive wollastonite/PHBV composite scaffolds, Biomaterials, 2004, 25, 5473-5480.

$4 \mathrm{~J} . \mathrm{Wu}, \mathrm{K}$. Xue, H. Li, J. Sun and K. Liu, Improvement of PHBV scaffolds with bioglass for cartilage tissue engineering, PLoS One, 2013, 8, e71563.

5 L.-C. Gerhardt and A. R. Boccaccini, Bioactive glass and glass-ceramic scaffolds for bone tissue engineering, Materials, 2010, 3, 3867-3910.

6 M. N. Rahaman, D. E. Day, B. S. Bal, Q. Fu, S. B. Jung, L. F. Bonewald, et al., Bioactive glass in tissue engineering, Acta Biomater., 2011, 7, 2355-2373.

7 Q. Y. Zeng, Y. Han, H. Y. Li and J. Chang, Design of a thermosensitive bioglass/agarose-alginate composite hydrogel for chronic wound healing, J. Mater. Chem. B, 2015, 3, 8856-8864.

8 H. W. Kim, J. H. Song and H. E. Kim, Bioactive glass nanofiber-collagen nanocomposite as a novel bone regeneration matrix, J. Biomed. Mater. Res., Part A, 2006, 79, 698-705.

9 P. Khoshakhlagh, S. M. Rabiee, G. Kiaee, P. Heidari, A. K. Miri, R. Moradi, et al., Development and characterization of a bioglass/chitosan composite as an injectable bone substitute, Carbohydr. Polym., 2017, 157, 1261-1271.

10 A. M. Haaparanta, P. Uppstu, M. Hannula, V. Ella, A. Rosling and M. Kellomaki, Improved dimensional stability with bioactive glass fibre skeleton in poly(lactide- $c o$-glycolide) porous scaffolds for tissue engineering, Mater. Sci. Eng., C, 2015, 56, 457-466.

$11 \mathrm{H}$. Li and J. Chang, Stimulation of proangiogenesis by calcium silicate bioactive ceramic, Acta Biomater., 2013, 9, 5379-5389.

$12 \mathrm{H}$. Li and J. Chang, Bioactive silicate materials stimulate angiogenesis in fibroblast and endothelial cell co-culture system through paracrine effect, Acta Biomater., 2013, 9, 6981-6991.
13 H. Li, K. Xue, N. Kong, K. Liu and J. Chang, Silicate bioceramics enhanced vascularization and osteogenesis through stimulating interactions between endothelia cells and bone marrow stromal cells, Biomaterials, 2014, 35, 3803-3818.

14 A. Leu, S. M. Stieger, P. Dayton, K. W. Ferrara and J. K. Leach, Angiogenic response to bioactive glass promotes bone healing in an irradiated calvarial defect, Tissue Eng., Part A, 2009, 15, 877-885.

15 A. Leu and J. K. Leach, Proangiogenic potential of a collagen/ bioactive glass substrate, Pharm. Res., 2008, 25, 1222-1229.

16 A. A. Gorustovich, J. A. Roether and A. R. Boccaccini, Effect of bioactive glasses on angiogenesis: a review of in vitro and in vivo evidences, Tissue Eng., Part B, 2010, 16, 199-207.

17 A. Hoppe, N. S. Guldal and A. R. Boccaccini, A review of the biological response to ionic dissolution products from bioactive glasses and glass-ceramics, Biomaterials, 2011, 32, 2757-2774.

18 M. W. Laschke, Y. Harder, M. Amon, I. Martin, J. Farhadi, A. Ring, et al., Angiogenesis in tissue engineering: breathing life into constructed tissue substitutes, Tissue Eng., Part A, 2006, 12, 2093-2104.

19 E. C. Novosel, C. Kleinhans and P. J. Kluger, Vascularization is the key challenge in tissue engineering, Adv. Drug Delivery Rev., 2011, 63, 300-311.

20 L. H. Nguyen, N. Annabi, M. Nikkhah, H. Bae, L. Binan, S. Park, et al., Vascularized bone tissue engineering: approaches for potential improvement, Tissue Eng., Part B, 2012, 18, 363-382.

21 B. Guillotin, R. Bareille, C. Bourget, L. Bordenave and J. Amedee, Interaction between human umbilical vein endothelial cells and human osteoprogenitors triggers pleiotropic effect that may support osteoblastic function, Bone, 2008, 42, 1080-1091.

22 M. Grellier, L. Bordenave and J. Amedee, Cell-to-cell communication between osteogenic and endothelial lineages: implications for tissue engineering, Trends Biotechnol., 2009, 27, 562-571.

23 S. Janardhanan, M. O. Wang and J. P. Fisher, Coculture strategies in bone tissue engineering: the impact of culture conditions on pluripotent stem cell populations, Tissue Eng., Part B, 2012, 18, 312-321.

24 C. J. Kirkpatrick, S. Fuchs and R. E. Unger, Co-culture systems for vascularization-learning from nature, $A d v$. Drug Delivery Rev., 2011, 63, 291-299.

25 K. Kyriakidou, G. Lucarini, A. Zizzi, E. Salvolini, M. Mattioli Belmonte, F. Mollica, et al., Dynamic co-seeding of osteoblast and endothelial cells on 3D polycaprolactone scaffolds for enhanced bone tissue engineering, $J$. Bioact. Compat. Polym., 2008, 23, 227-243.

26 K. G. Battiston, J. W. Cheung, D. Jain and J. P. Santerre, Biomaterials in co-culture systems: towards optimizing tissue integration and cell signaling within scaffolds, Biomaterials, 2014, 35, 4465-4476.

$27 \mathrm{H}$. Li and J. Chang, pH-compensation effect of bioactive inorganic fillers on acidic degradation products of PLGA, Compos. Sci. Technol., 2005, 65, 2226-2232. 
28 E. A. Jaffe, Culture of human endothelial cells, Transplant. Proc., 1980, 12, 49-53.

29 S. K. Levengood, M. J. Poellmann, S. G. Clark, D. A. Ingram, M. C. Yoder and A. J. Johnson, Human endothelial colony forming cells undergo vasculogenesis within biphasic calcium phosphate bone tissue engineering constructs, Acta Biomater., 2011, 7, 4222-4228.

30 N. Sultana and M. Wang, Fabrication of HA/PHBV composite scaffolds through the emulsion freezing/freeze-drying process and characterisation of the scaffolds, J. Mater. Sci.: Mater. Med., 2008, 19, 2555-2561.

31 N. Goonoo, A. Bhaw-Luximon, P. Passanha, S. R. Esteves and D. Jhurry, Third generation poly(hydroxyacid) composite scaffolds for tissue engineering, J. Biomed. Mater. Res., Part $B, 2016$, DOI: $10.1002 / \mathrm{jbm}$. b.33674.

$32 \mathrm{H}$. Li, W. Zhai and J. Chang, Effects of wollastonite on proliferation and differentiation of human bone marrow- derived stromal cells in PHBV/wollastonite composite scaffolds, J. Biomater. Appl., 2009, 24, 231-246.

33 V. I. Macias-Andres, W. Li, E. A. Aguilar-Reyes, Y. Ding, J. A. Roether, L. Harhaus, et al., Preparation and Characterization of $45 \mathrm{~S} 5$ Bioactive Glass-based Scaffolds Loaded with PHBV Microspheres with Daidzein Release Function, J. Biomed. Mater. Res., Part A, 2017, DOI: 10.1002/ jbm.a.36046.

34 H. Li, J. He, H. Yu, C. R. Green and J. Chang, Bioglass promotes wound healing by affecting gap junction connexin 43 mediated endothelial cell behavior, Biomaterials, 2016, 84, 64-75.

35 X. Zhao, L. Liu, F. K. Wang, D. P. Zhao, X. M. Dai and X. S. Han, Coculture of vascular endothelial cells and adipose-derived stem cells as a source for bone engineering, Ann. Plast. Surg., 2012, 69, 91-98.

36 G. I. Im, Coculture in musculoskeletal tissue regeneration, Tissue Eng., Part B, 2014, 20, 545-554. 